\title{
Ultrafast Electron Diffraction and Microscopy: Why We Should Know About Transient Electric Fields and Where They Come From
}

\author{
Hyuk Park ${ }^{1,2}$ and Jian-Min Zuo ${ }^{1,2}$ \\ ${ }^{1}$ Department of Materials Science and Engineering, University of Illinois, Urbana-Champaign, \\ Urbana, IL 61801 USA \\ ${ }^{2}$ Frederick Seitz Materials Research Laboratory, University of Illinois, Urbana-Champaign, Urbana, \\ IL 61801 USA
}

Developments in ultrafast lasers and pulsed electron gun have led to the development of timeresolved electron diffraction and microscopy with temporal resolution approaching tenths of picoseconds $(\mathrm{ps})^{1-4}$. The combination of ultrafast laser and reflection high-energy electron diffraction (RHEED), for example, provides a possibility to examine the surface dynamics at the atomic time and length scales. A number of publications, including in high profile journals, reported large lattice contraction and expansion and phase transitions in several materials on the time scale of few picoseconds (ps) to tens or hundreds of ps using ultrafast RHEED ${ }^{5-6}$. Repeatable processes at ps time scale have also been reported by ultrafast $\mathrm{EM}^{4}$. Ultrafast electron diffraction and microscopy use pulsed laser as pump to initiate dynamic processes in solids. Under irradiation of pulsed laser beam of picoseconds or less, electrons inside a solid can be heated to high temperatures for a short period of time (several ps). A part of hot electrons can be emitted from the surface in a similar way of thermionic emission ${ }^{7}$. The emitted electrons, travel at speeds, produce transient electric fields (TEFs) together with the positively charged surface ${ }^{8}$. However, previous reports did not take account of photoemitted electrons and their electric fields, and thus ignored their effect on both the incident and diffracted electron beams.

To measure TEFs, we have developed a time-resolved electron beam imaging technique based on the pump-probe approach. We monitor a small diameter of collimated electron pulses traveling above the targeted surface as a function of delay time between pump laser $(800 \mathrm{~nm})$ and probe electron beam $(30 \mathrm{kV})$. The experimental setup used for detecting transient electrical fields has been described in detail elsewhere ${ }^{8}$. Figure 1a shows selected images of the electron beam recorded at different time delays for the laser fluence of $67.7 \mathrm{~mJ} / \mathrm{cm}^{2}$ above a silicon surface. To monitor the relative change we took the intensity profile of the direct electron beam along the vertical direction averaged over a horizontal width of the direct beam and combine the profiles for each time delay to form a composite image of intensity versus the time delay (horizontal axis) as shown in Figure $1 \mathrm{~b}$. The sample is at the bottom of each image. At $75 \mathrm{ps,} \mathrm{the} \mathrm{electron} \mathrm{beam} \mathrm{is} \mathrm{deflected} \mathrm{upper} \mathrm{ward} \mathrm{with}$ a narrower beam width compared to that at 0 ps. Afterward, the electron beam moved downward (toward the surface) with a larger beam width. The position of electron beam is not fully recovered to the original position within $1 \mathrm{~ns}$ delay time.

To explain the time dependence of electron beam deflection, we developed a model of the electron plume produced by the laser pulse and its propagation ${ }^{8}$. The model assumed that emitted electrons leave the sample surface and propagate at a velocity of $v_{\mathrm{o}}$. For the propagation of electrons, we assume a part is subjected to a de-acceleration, while the rest continues at its initial velocity. Using the above model, we fitted the measured time dependent electron beam deflections. The results were reported in ref. ${ }^{8}$. Figure 2 shows the time dependent electron (charge) distribution $\rho(z, t)$ obtained from fitting for the laser fluence of $67.7 \mathrm{~mJ} / \mathrm{cm}^{2}$ at several time delays. 
In ultrafast RHEED, TEFs lead to deflection of specular and diffracted electron beams, which previously was explained entirely based on lattice contraction and expansion ${ }^{5-6}$. In ultrafast electron microscopy, TEFs from accidental laser irradiation of supporting metal grids can lead to anomalous beam deflection and change in image contrast. Thus, there is clearly a need for better understanding of TEFs in the field of ultrafast electron microscopy. The work is supported by DOE DEFG0201ER4592, DEFG02-91-ER45439 and DOE DEFG02-07ER46453.

\section{Reference:}

${ }^{1}$ G. Mourou and S. Williamson, Applied Physics Letters 41, 44 (1982).

${ }^{2}$ J. Cao et al., Applied Physics Letters 83, 1044 (2003).

${ }^{3}$ B. J. Siwick et al., Journal of Applied Physics 92, 1643 (2002).

${ }^{4}$ B. Barwick, H. S. Park, O. H. Kwon, J. S. Baskin, and A. H. Zewail, Science 322, 1227 (2008).

${ }^{5}$ C. Y. Ruan, V. A. Lobastov, F. Vigliotti, S. Y. Chen, and A. H. Zewail, Science 304, 80 (2004).

${ }^{6}$ C. Y. Ruan, F. Vigliotti, V. A. Lobastov, S. Y. Chen, and A. H. Zewail, PNAS 101, 1123 (2004).

${ }^{7}$ J. G. Fujimoto, J. M. Liu, E. P. Ippen, and N. Bloembergen, Phy. Rev. Let. 53, 1837 (1984).

${ }^{8}$ H. Park and J. M. Zuo, Applied Physics Letters 94, 251103 (2009).
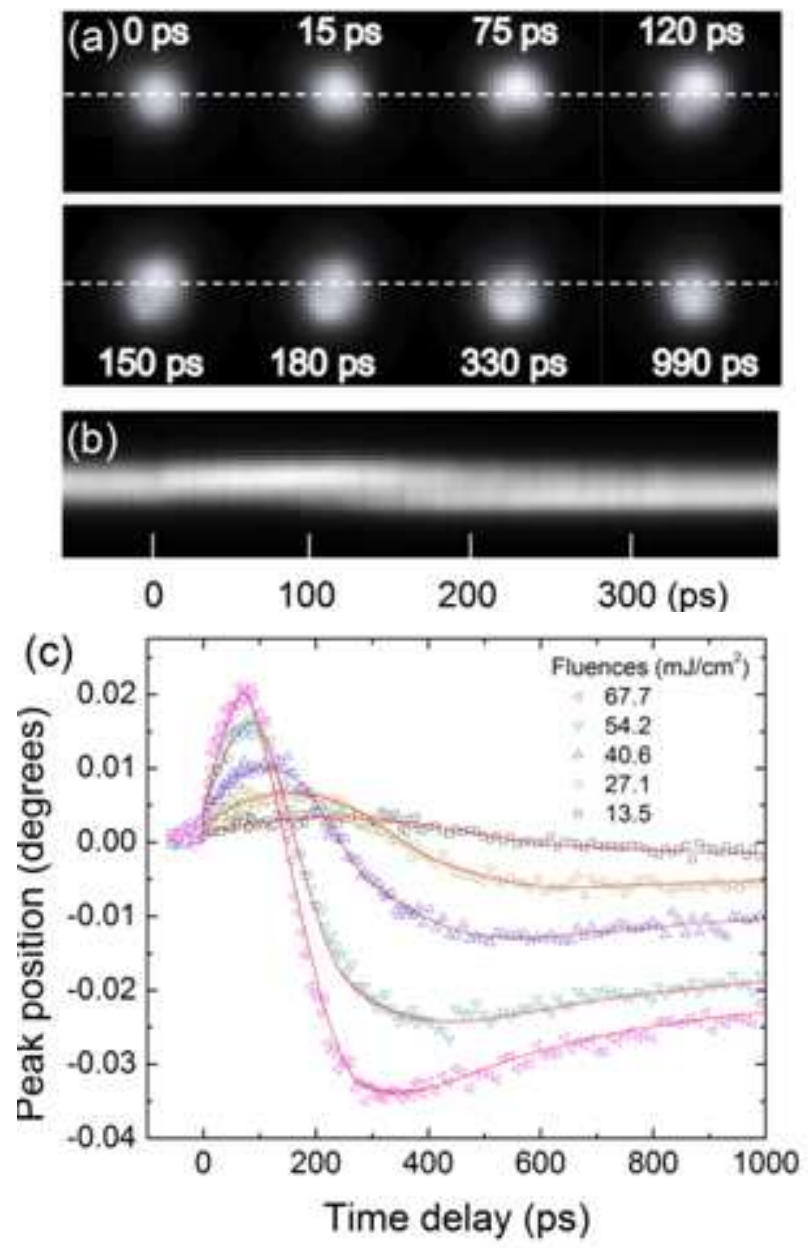

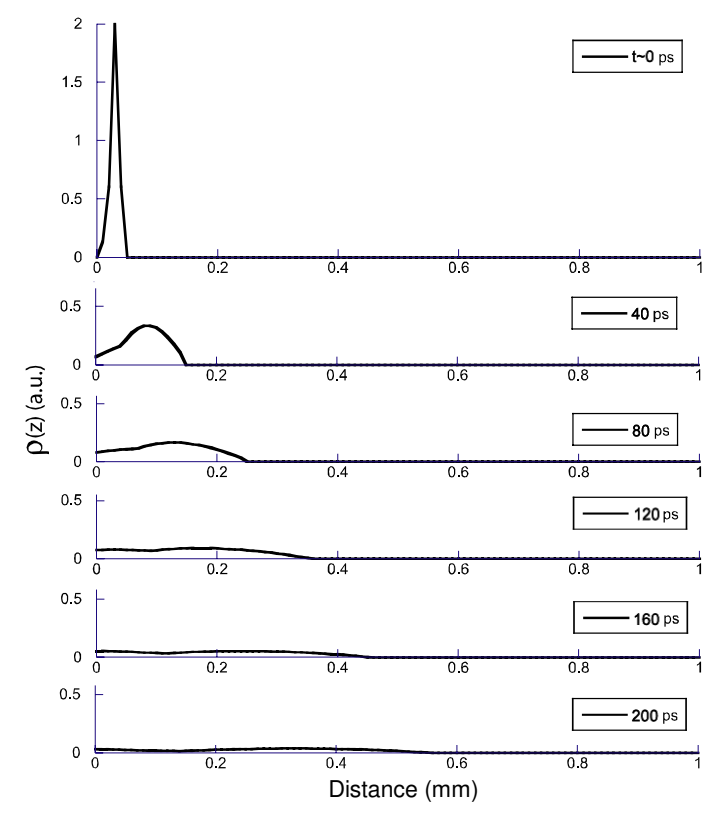

Figure 2 Time dependent electron (charge) distribution $\rho(z, t)$ obtained from fitting for the laser fluence of $67.7 \mathrm{~mJ} / \mathrm{cm}^{2}$ at several time delays

Figure 1. (a) Temporal evolution of electron shadow image with the laser fluence of 67.7 $\mathrm{mJ} / \mathrm{cm}^{2}$. The dash line indicates the initial electron beam position. (b) Composite image of the vertical electron probe intensity profile (vertical) as a function of the delay (horizontal). (c) Vertical electron beam deflection as a function of delay time in degrees. The continuous lines are results of model fitting based on TEFs. 